PROCEEDINGS OF THE

AMERICAN MATHEMATICAL SOCIETY

Volume 98, Number 4, December 1986

\title{
ON THE STABLE RANGE OF UNIFORM ALGEBRAS AND $H^{\infty}$
}

\author{
GUSTAVO CORACH AND FERNANDO DANIEL SUÁREZ
}

\begin{abstract}
We find a criterion for stable range reducibility for uniform algebras and we apply it to the algebra $H^{\infty}$.
\end{abstract}

The notion of stable rank of a ring introduced by Bass [1] , when applied to a commutative Banach algebra $A$, is strongly related with some homotopical invariants of its spectrum $X(A)$ and of the zero-sets of its ideals [2, 3]. An $n$-tuple $a \in A^{n}$ is unimodular if $A=\sum_{i=1}^{n} A a_{i} ; a$ is reducible if $\left(a_{1}+x_{1} a_{n}, \ldots, a_{n-1}+x_{n-1} a_{n}\right)$ is unimodular for some $x_{1}, \ldots, x_{n-1} \in A$. The stable rank of $A$ is the least $n$ such that every unimodular $a \in A^{n+1}$ is reducible.

In [3] we conjectured that the algebra $H^{\infty}$ of all bounded holomorphic functions on the open unit disc $D$ has stable rank one, i.e. that for every $f, g \in H^{\infty}$ such that $|f|+|g| \geq \delta_{1}$ for some $\delta_{1}>0$, there exists $h \in H^{\infty}$ with $|f+h g| \geq \delta_{2}$ for some $\delta_{2}>0$. The problem is still open. In this note, however, we prove that every unimodular $(f, g)$, with $f$ in the disc algebra, is reducible. The proof of this result (Theorem 2) is based on some work of D. J. Newman, used by L. Carleson for proving his corona theorem, and on a result (Theorem 1) which asserts that if $A$ is a uniform algebra and $\alpha, \beta \in A$ are such that $\left(\alpha-z_{i}, \beta\right)$ is reducible for suitable complex numbers $z_{i}$, then every unimodular of the form $(F(\alpha), \beta)$, where $F \in R(\operatorname{sp} \alpha)$, is reducible (recall that if $K$ is a compact subset of the plane, $R(K)$ is the algebra of uniform limits of rational functions with poles outside $K$ ).

NOTATIONS. Given a complex commutative Banach algebra, the spectrum $X(A)$ is the space of all nonzero homomorphisms $A \rightarrow \mathbf{C}$ with the topology of simple convergence; it is a compact Hausdorff space and the Gelfand transform $\hat{a}$ of an element $a$ of $A$ is the continuous function $\hat{a}: X(A) \rightarrow \mathbf{C}$ defined by $\hat{a}(h)=h(a)(h \in$ $X(A))$. The image of $\hat{a}$ coincides with the spectrum of $a, \operatorname{sp} a=\{\lambda \in \mathbf{C} ; a-\lambda$ is not invertible $\}$. We shall use several times the notation $Z_{a}$ for the closed set $\{h \in X(A)$; $h(a)=0\}$. Throughout this paper $D$ denotes the open unit disc $\{z \in \mathbf{C} ;|z|<1\}$. $H^{\infty}$ is the algebra of all bounded holomorphic functions on $D$ with the sup norm. Its spectrum is not known (see Hoffman [5] for some of the known facts about it); given $w \in T=\{z \in \mathbf{C} ;|z|=1\}, M_{w}=\left\{h \in X\left(H^{\infty}\right) ; h(z)=w\right\}$ (where $z$ is the coordinate function).

Let $A$ be a commutative ring with identity. An element $(\alpha, \beta)$ of $A^{2}$ is unimodular if $A \alpha+A \beta=A ; U_{2}(A)$ is the set of all unimodular elements of $A^{2}$. We say that $(\alpha, \beta) \in U_{2}(A)$ is reducible (in the sense of Bass) if there exists $\gamma \in A$ such that $\alpha+\gamma \beta$ is invertible. For example, if $\alpha$ is invertible, $(\alpha, \beta)$ is unimodular and

Received by the editors April 1, 1985 and, in revised form, November 13, 1985.

1980 Mathematics Subject Classification (1985 Revision). Primary 46J15, 42B30, 30H05; Secondary 18F25. 
reducible for every $\beta \in A$ (take $\gamma=0$ ). It is easy to prove that

(1) $(\alpha, \beta) \in U_{2}(A)$ is reducible if and only if $\alpha \in A+A \beta$ ( $A$ is the group of invertible elements of $A$ );

(2) if $(\alpha, \beta),\left(\alpha^{\prime}, \beta\right) \in U_{2}(A)$ are reducible, then $\left(\alpha \alpha^{\prime}, \beta\right) \in U_{2}(A)$, and $\left(\alpha \alpha^{\prime}, \beta\right)$ is reducible, too.

PROPOSITION. Let $A$ be a commutative Banach algebra with identity. For every $\beta \in A$ let $H_{1}(A ; \beta)=\left\{\alpha \in A ;(\alpha, \beta) \in U_{2}(A)\right\}$. Then $A+A \beta$ is open and closed in $H_{1}(A ; \alpha)$. In particular, if $f:[0,1] \rightarrow H_{1}(A ; \beta)$ is a continuous curve and $(f(0), \beta)$ is reducible, then $(f(1), \beta)$ is reducible, too.

PROOF. It is clear that $A+A \beta$ and $H_{1}(A ; \beta)$ are open in $A^{2}$. So it suffices to prove that $A+A \beta$ is closed in $H_{1}(A ; \beta)$. Let $u_{n} \in A, x_{n} \in A$ such that $u_{n}+x_{n} \beta$ converges to $\alpha \in H_{1}(A ; \beta)$. Then there exist $a, b \in A$ such that $a \alpha+b \beta=1$. Now, $A^{\prime}$ is open, so $a\left(u_{n}+x_{n} \beta\right)+b \beta \in A^{\prime}$ for $n$ large enough, so $a=u+x \beta$ for some $u \in A$ and $x \in A$; thus, $1=a \alpha+b \beta=u \alpha+x \alpha \beta+b \beta=u \alpha+(x \alpha+b) \beta$ and $\alpha=u^{-1}-u^{-1}(x \alpha+b) \beta \in A+A \beta$.

REMARKS. For each positive integer, $U_{n}(A)$ can be defined as the set of all $a=\left(a_{1}, \ldots, a_{n}\right) \in A^{n}$ such that $\sum_{i=1}^{n} A a_{i}=A$. There is also an $n$-dimensional version of reducibility which leads to the stable range conditions of Bass [1]. For commutative rings the statements (1) and (2) above hold replacing $U_{2}(A)$ by $U_{n+1}(A)$, $A$ by $U_{n}(A)$, and $A$ by $A^{n}$. The proposition can be generalized, too (see Corach and Suárez [3, Lemma 3.7]), but we do not know if $U_{n}(A)+A^{n} \alpha$ is closed in $H_{n}(A ; \alpha)$ when $A$ is noncommutative. If this happens, several facts on the stable rank of Banach algebras, which hold in the commutative case, could be generalized (see Corach and Larotonda [2] and Corach and Suárez [3]).

THEOREM 1. Let $A$ be a uniform algebra and $\alpha, \beta \in A$. Let $D_{i}, i \in I$, be the connected components of $\mathbf{C} \backslash \hat{\alpha}\left(Z_{\beta}\right)$. Suppose that, for each $i \in I$, there exists $z_{i} \in D_{i}$ such that $\left(\alpha-z_{i}, \beta\right) \in U_{2}(A)$ is reducible. Then for every $F \in R(\operatorname{sp} \alpha)$ such that $(F(\alpha), \beta) \in U_{2}(A)$ it holds that $(F(\alpha), \beta)$ is reducible.

PROOF. For every $z \in \mathbf{C} \backslash \hat{\alpha}\left(Z_{\beta}\right),(\alpha-z, \beta)$ is reducible. In fact, if $\gamma:[0,1] \rightarrow$ $D_{i} \subset \mathbf{C} \backslash \hat{\alpha}\left(Z_{\beta}\right)$ is an arc joining $z_{i}$ and $z$, it is clear that $(\alpha-\gamma(t), \beta) \in U_{2}(A) \forall t \in$ $[0,1]$, so $\alpha-z_{i}$ and $\alpha-z$ belong to the same component of $H_{1}(A ; \alpha)$.

Thus, for every $z \in \mathbf{C} \backslash \hat{\alpha}\left(Z_{\beta}\right)$ there exists $\mu \in A$ such that $\hat{\alpha}-z=\hat{\mu}$ on $Z_{\beta}$.

Let $(F(\alpha), \beta) \in U_{2}(A)$ for some $F \in R(\operatorname{sp} \alpha)$. Then $Z_{F} \cap \hat{\alpha}\left(Z_{\beta}\right)=\varnothing$, for $(F(\alpha), \beta) \in U_{2}(A)$ means that $Z_{F(\alpha)} \cap Z_{\beta}=\varnothing$. In particular, we can find rational functions $f_{n}$ with poles outside $\operatorname{sp} \alpha$ and zeros outside $\hat{\alpha}\left(Z_{\beta}\right)$ such that $\left\{f_{n}\right\}$ converges uniformly to $F$ on $\operatorname{sp} \alpha$. Then $f_{n}(\alpha) \rightarrow F(\alpha)$ and $\left(f_{n}(\alpha), \beta\right)$ is reducible, or, what is the same, $f_{n}(\alpha) \in A+A \beta$, because $f_{n}(\alpha)$ is a product of elements which are reducible with $\beta$. But $A+A \beta$ is closed in $H_{1}(A ; \beta)$ by the proposition, so $F(\alpha) \in A^{\cdot}+A \beta$ and $(F(\alpha), \beta)$ is reducible.

COROLLARY 1. The same conclusion of Theorem 1 holds if every component of $\mathbf{C} \backslash \hat{\alpha}\left(Z_{\beta}\right)$ contains an element of $\mathbf{C} \backslash \operatorname{sp} \alpha$.

Proof. For every component $D_{i}$ of $\mathbf{C} \backslash \hat{\alpha}\left(Z_{\beta}\right)$, let $z_{i} \in(\mathbf{C} \backslash \operatorname{sp} \alpha) \cap D_{i}$. Then $\alpha-z_{i} \in A$ and $\left(\alpha-z_{i}, \beta\right) \in U_{2}(A)$ must be reducible. Thus the hypotheses of Theorem 1 are satisfied. 
COROLlaRY 2. Let $(\alpha, \beta) \in U_{2}(A)$ and suppose that $\mathbf{C} \backslash \hat{\alpha}\left(Z_{\beta}\right)$ is connected. Then $(\alpha, \beta)$ is reducible.

PROOF. In view of Theorem 1, taking $F(w)=w \forall w$ it suffices to exhibit $z \in \mathbf{C} \backslash \hat{\alpha}\left(Z_{\beta}\right)$ with $(\alpha-z, \beta)$ reducible. For this, take $z=\|\alpha\|+1$; then $z \in$ $\mathbf{C} \backslash \operatorname{im} \hat{\alpha} \subset \mathbf{C} \backslash \hat{\alpha}\left(Z_{\beta}\right)$ and $\alpha-z \in A$, in particular, $(\alpha-z, \beta)$ is reducible.

In [3] it was conjectured that $H^{\infty}$ has stable rank one, or what is the same, that every $(f, g) \in U_{2}\left(H^{\infty}\right)$ is reducible. As an approximation to the proof of this conjecture, we have the following result.

THEOREM 2. Let $(f, g) \in U_{2}\left(H^{\infty}\right)$. Suppose that $f$ belongs to the disc algebra. Then $(f, g)$ is reducible.

The proof is largely based on the corona theorem of L. Carleson, particularly on a result due to D. J. Newman, as it appears in [4, Chapter 12]. More precisely, we need the following two lemmas [4, pp. 203-205].

LEMMA A. Let $B(z)=\prod_{k=1}^{s}\left(z-a_{k}\right) /\left(1-\bar{a}_{k} z\right)$ be a finite Blaschke product with distinct zeros $a_{1}, \ldots, a_{s}$. For $\delta<1 / 2$ let $F$ be analytic in the set $\{z ;|B(z)|<\delta\}$ and satisfy $|F(z)|<1$ there. Then there exists $f \in H^{\infty}$ with $f\left(a_{k}\right)=F\left(a_{k}\right)$, $k=1, \ldots, s$ and $\|f\| \leq \delta^{-\alpha}$, where $\alpha$ is an absolute constant.

LEMMA B. Let $f$ be a function in the disc algebra such that $0<|f(z)| \leq 1$ on $|z|=1$ and $E=\{z ;|z|=1,|f(z)|<1\}$ is nonempty. Then there exists $a$ sequence $\left\{B_{n}\right\}$ of finite Blaschke products with simple zeros such that $\left|B_{n}(z)\right| \rightarrow$ $|f(z)|$ uniformly on each closed subset of $\bar{D} \backslash \bar{E}$ and $B_{n}(z) \rightarrow f(z)$ uniformly on each closed subset of $D$.

PROOF OF THEOREM 2. Using a Möbius transformation we may suppose that $g(0) \neq 0$ and $\|g\|=1$. It follows from Theorem 1 that it suffices to prove that $(z, g)$ is reducible; in fact, $\hat{z}\left(Z_{g}\right)=\left\{w \in S^{1}\right.$; there exists $\phi \in M_{w}$ such that $\phi(g)=0\} \cup\{w \in D ; g(w)=0\}$, so $\mathbf{C} \backslash \hat{z}\left(Z_{g}\right)$ has one or two connected components; in the first case, we use Corollary 2 of Theorem 1 ; in the second case, $(z-w, g)$ is reducible if $|w|>1$ because $z-w$ is invertible, so if we prove that $(z, g)$ is reducible we apply Theorem 1 , observing that $R(\operatorname{sp} \hat{z})$ is the disc algebra.

Let $0<\delta_{0}<1$ such that $|z|+|g(z)|>\delta_{0}$ for every $z \in D$. There exists $E \subset S^{1}=\{z ;|z|=1\}$ with measure $2 \pi$ such that $g$ has nontangential limit at every $z \in E$; the limit function will be denoted by $g$, too.

Let $V=\left\{z_{0} /\left|z_{0}\right| ; z_{0} \in D, g\left(z_{0}\right)=0\right\} \cup\{z \in E ; g(z)=0\}$. Then $E \backslash V$ has measure $2 \pi$. If $z_{1} \in E \backslash V$ there exists $\delta_{1}>0$ such that $\left|g\left(r z_{1}\right)\right|>\delta_{1}$ for $0 \leq r<1$. Let $R=\left\{r z_{1} ; 0 \leq r<1\right\}$ and $\delta=\min \left\{\delta_{0}, \delta_{1}\right\}$. Then $|z|+|g(z)|>\delta$ for every $z \in D$ and $|g(z)|>\delta$ for every $z \in R$.

For $h \in H^{\infty}$ let $S_{\delta}(h)=\{z \in D ;|h(z)|<\delta / 2\}$. Consider $g_{\rho}(z)=g(\rho z)$ for those $\rho<1$ such that $g(\rho z) \neq 0$ in $S^{1}$. Then $R \subset D \backslash S_{\delta}\left(g_{\rho}\right)$ and $|z|+\left|g_{\rho}(z)\right|>\delta$, for $|z|+\left|g_{\rho}(z)\right|>|\rho z|+\left|g_{\rho}(z)\right|>\delta$ if $z \in D$. Let $\phi_{\rho}$ be a nonvanishing function in the disc algebra such that $\left|\phi_{\rho}(z)\right|=\min \left\{3 / \delta, 1 /\left|g_{\rho}(z)\right|\right\}$ for $z \in S^{1}$, and let $\psi_{\rho}(z)=\phi_{\rho}(z) g_{\rho}(z)$. Observe that $\phi_{\rho}$ can be constructed by means of a Poisson integral and an exponential.

Then the following facts hold:

(i) $\left|\phi_{\rho}(z)\right| \geq 1$ and $\left|\psi_{\rho}(z)\right| \leq 1$ for every $z \in \bar{D}$, 
(ii) for $z \in S^{1},\left|\psi_{\rho}(z)\right|=1 \Leftrightarrow\left|\phi_{\rho}(z)\right|=1 /\left|g_{\rho}(z)\right| \Leftrightarrow\left|g_{\rho}(z)\right| \geq \delta / 3$,

(iii) for each $\rho$ there exists a sequence $B_{n}$ of finite Blaschke products with simple zeros such that $B_{n} \rightarrow \psi_{\rho}$ uniformly on the compacts of $D$, and $\left|B_{n}(z)\right|>\left|\psi_{\rho}(z)\right|-$ $\delta / 4$ for $n$ sufficiently large if $\left|g_{\rho}(z)\right| \geq \delta / 2$ (Lemma B),

(iv) $|z|+\left|B_{n}(z)\right|>\delta / 2$ for $z \in D$ (in fact, $|z|+\left|B_{n}(z)\right|>|z|+\left|\psi_{\rho}(z)\right|-\delta / 4 \geq$ $|z|+\left|g_{\rho}(z)\right|-\delta / 4>\delta / 2$ for $\left|g_{\rho}(z)\right| \geq \delta / 2$, and $|z|>\delta / 2$ for $\left.\left|g_{\rho}(z)\right|<\delta / 2\right)$,

(v) $\left|B_{n}(z)\right| \geq \delta / 4$ for every $z \in R$ (for $R \subset\left\{z ;\left|g_{\rho}(z)\right| \geq \delta / 2\right\}$ and, by (iii), $\left.\left|B_{n}(z)\right|>\left|\psi_{\rho}(z)\right|-\delta / 4 \geq\left|g_{\rho}(z)\right|-\delta / 4 \geq \delta / 4\right)$.

Fixing $\rho$ and $n$ we have $\left|B_{n}(z)\right|+|z|>\delta / 2$ for every $z$, and $\left|B_{n}(z)\right| \geq \delta / 4$ in $R$; then $S_{\delta / 2}\left(B_{n}\right)=\bigcup_{i \in I} D_{i}$, where each $D_{i}$ is a connected component, which is simply connected by the maximum modulus principle; then there exists a logarithmic branch $\ln z$ on $D \backslash R$, such that, putting $\ln _{i} z=\ln z$ for $z \in D_{i}$ we have $\left|\ln _{i} z\right| \leq$ $T_{\delta}=\left[(\ln \delta / 4)^{2}+(2 \pi)^{2}\right]^{1 / 2}$ (observe that $|z| \geq \delta / 4$ in $S_{\delta / 2}\left(B_{n}\right)$ ), so by Lemma A, there exists $r_{n} \in H^{\infty}$ such that $r_{n}\left(a_{k}^{i}\right)=\ln _{i} a_{k}^{i}$ for every $a_{k}^{i} \in Z_{B_{n}} \cap D_{i}$ and $\left\|r_{n}\right\| \leq s=T_{\delta}(\delta / 4)^{-\alpha}$, where $\alpha$ is an absolute constant. Then $z-e^{r_{n}}$ vanishes at every $a_{k}^{i}$, so there exists $h_{n} \in H^{\infty}$ such that $z-e^{r_{n}(z)}=h_{n}(z) B_{n}(z)$; then $\left|h_{n}(z)\right|=\left|z-e^{r_{n}(z)}\right|$ a.e. in $S^{1}$ in the sense of the nontangential limit function, and $\left\|h_{n}\right\| \leq 1+e^{\left\|r_{n}\right\|} \leq 1+e^{s}$. This shows that $\left\{r_{n}\right\}$ and $\left\{h_{n}\right\}$ are normal families $(\rho$ remains fixed), and taking subsequences we have $h_{n} \rightarrow h_{\rho}$ and $r_{n} \rightarrow r_{\rho}$ uniformly on the compacts of $D$, for some $h_{\rho}$ and $r_{\rho}$ in $H^{\infty}$. Moreover, $\left\|h_{\rho}\right\| \leq 1+e^{s}$, $\left\|r_{\rho}\right\| \leq s$ and $z-h_{\rho}(z) \phi_{\rho}(z) g_{\rho}(z)=e^{r_{\rho}(z)}$. By the definition of $\phi_{\rho},\left\|\phi_{\rho}\right\| \leq 3 / \delta$. These three inequalities show that $\left\{\phi_{\rho}\right\},\left\{h_{\rho}\right\},\left\{r_{\rho}\right\}$ are normal families and, taking $\rho \rightarrow 1$ with $g_{\rho}(z) \neq 0$ in $S^{1}$ through a suitable sequence, we get $h_{\rho} \rightarrow h, \phi_{\rho} \rightarrow \phi$, $r_{\rho} \rightarrow r$ and $g_{\rho} \rightarrow g$ uniformly on the compacts of $D$ for some $h, \phi, r$ in $H^{\infty}$ with $\|h\| \leq 1+e^{s},\|r\| \leq s,\|\phi\| \leq 3 / \delta$, and $z-h(z) \phi(z) g(z)=e^{r(z)}$. This shows that $(z, g)$ is reducible, as desired.

REMARK. We have proved, indeed, that $(f, g)$ is reducible to an element of $H^{\infty}$ which is in the connected component of the neutral element.

\section{REFERENCES}

1. H. Bass, K-theory and stable algebra, Inst. Hautes Études Sci. Publ. Math. 22 (1964), 5-60.

2. G. Corach and A. R. Larotonda, Stable range in Banach algebras, J. Pure Appl. Algebra 32 (1984), 289-300.

3. G. Corach and F. D. Suárez, Stable range in holomorphic function algebras, Illinois J. Math. 29 (1985), 627-639.

4. P. Duren, Theory of $H^{p}$ spaces, Academic Press, New York, 1970.

5. K. Hoffman, Banach spaces of analytic functions, Prentice Hall, Englewood Cliffs, N. J., 1962.

6. P. Jones, D. Marshall and T. Wolff, Stable rank of the disc algebra, Proc. Amer. Math. Soc. 96 (1986), 603-604.

Instituto Argentino de matemática, Viamonte 1636, $2^{\circ}$ C, Buenos Aires, ARGENTINA 